\title{
TRITIUM RECYCLING AND INVENTORY IN ERODED DEBRIS \\ OF PLASMA-FACING MATERIALS*
}

\author{
Ahmed Hassanein \\ Argonne National Laboratory, USA
}

The submitted manuscript has been created by the

University of Chicago as Operator of Argonne

National Laboratory ("Argonne") under Contract No.

W-31-109-ENG-38 with the U.S. Department of

Energy. The U.S. Government retains for itself, and

others acting on its behalf, a paid-up, nonexclusive,

irrevocable worldwide license in said article to

reproduce, prepare derivative works, distribute copies

to the public, and perform publicly and display

publicly, by or on behalf of the Government.

Invited Paper at Advanced Research Workshop on

HYDROGEN RECYCLE AT PLASMA FACING MATERIALS

Sept. 15-17, 1999, St. Petersburg, Russia

*Work is supported by the U.S. Department of Energy, Office of Fusion Energy

Science, under Contract W-31-109-Eng-38. 


\section{DISCLAIMER}

This report was prepared as an account of work sponsored by an agency of the United States Government. Neither the United States Government nor any agency thereof, nor any of their employees, make any warranty, express or implied, or assumes any legal liability or responsibility for the accuracy, completeness, or usefulness of any information, apparatus, product, or process disclosed, or represents that its use would not infringe privately owned rights. Reference herein to any specific commercial product, process, or service by trade name, trademark, manufacturer, or otherwise does not necessarily constitute or imply its endorsement, recommendation, or favoring by the United States Government or any agency thereof. The views and opinions of authors expressed herein do not necessarily state or reflect those of the United States Government or any agency thereof. 


\section{DISCLAIMER}

Portions of this document may be illegible in electronic image products. Images are produced from the best available original document. 


\title{
TRITIUM RECYCLING AND INVENTORY IN ERODED DEBRIS OF PLASMA-FACING MATERIALS
}

\author{
AHMED HASSANEIN \\ Argonne National Laboratory \\ Argonne, IL 60439, USA
}

\section{Abstract}

Damage to plasma-facing components (PFCs) and structural materials due to loss of plasma confinement in magnetic fusion reactors remains one of the most serious concerns for safe, successful, and reliable tokamak operation. High erosion losses due to surface vaporization, spallation, and melt-layer splashing are expected during such an event. The eroded debris and dust of the PFCs, including trapped tritium, will be contained on the walls or within the reactor chamber; therefore, they can significantly influence plasma behavior and tritium inventory during subsequent operations.

Tritium containment and behavior in PFCs and in the dust and debris is an important factor in evaluating and choosing the ideal plasma-facing materials (PFMs). Tritium buildup and release in the debris of candidate materials is influenced by the effect of material porosity on diffusion and retention processes. These processes have strong nonlinear behavior due to temperature, solubility, and existing trap sites. A realistic model must therefore account for the nonlinear and multidimensional effects of tritium diffusion in the porous-redeposited and neutron-irradiated materials. A tritium-transport computer model, TRAPS (TRitium Accumulation in Rorous Structure), was developed and used to evaluate and predict the kinetics of tritium transport in porous media. This 
model is coupled with the TRICS (Tritium In Compound Systems) code that was developed to study the effect of surface erosion during normal and abnormal operations on tritium behavior in PFCs.

\section{Introduction}

Damage to plasma-facing and nearby components as a result of various plasma instabilities that cause loss of plasma confinement remains a serious obstacle to successful tokamak operation. Plasma instabilities take various forms such as hard disruptions, which include both thermal and current quench (sometimes producing runaway electrons), edge-localized modes (ELMs), and vertical displacement events (VDEs). Plasma instabilities can cause surface and bulk damage to plasma-facing and structural materials. Surface damage includes high erosion losses from surface vaporization, spallation, and melt-layer erosion. In addition to these effects, the transport and redeposition of the eroded surface materials to various locations on plasma-facing and nearby components are of major concern for plasma contamination, for safety (dust inventory hazard), and for successful and prolonged plasma operation following instability events [1]. Figure 1 schematically illustrates possible erosion products and effects of plasma instabilities on target surface materials.

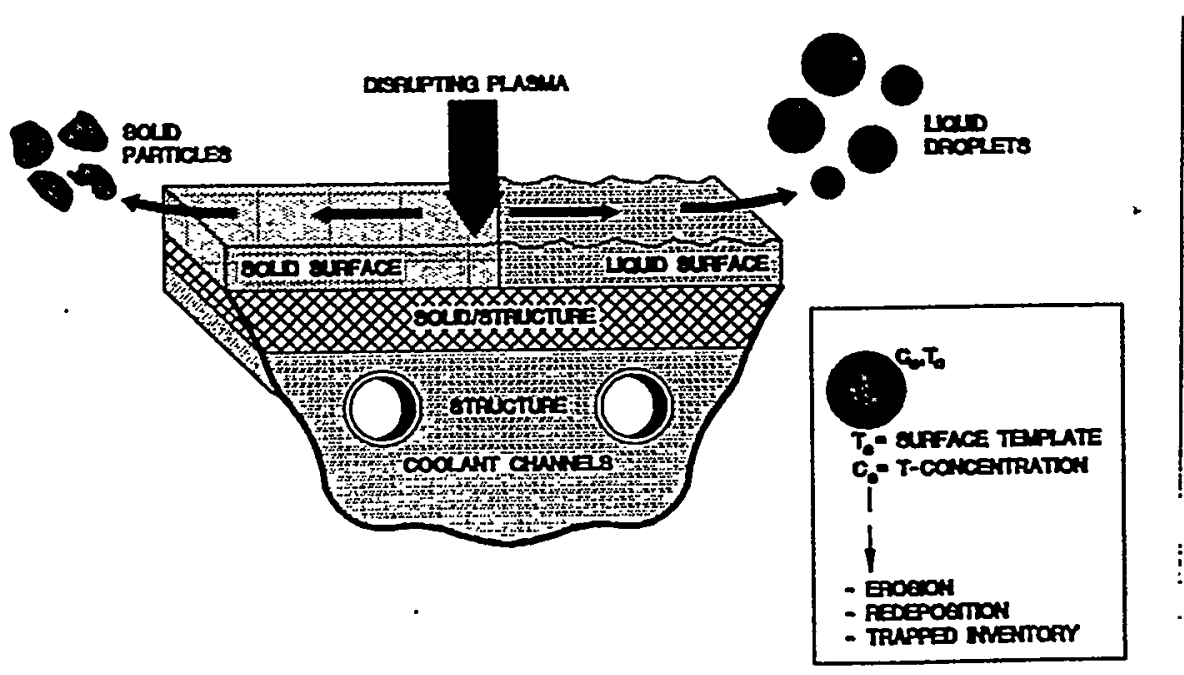

Fig. 1. Schematic illustration of erosion products as a result of plasma instabilities. 
Four key factors are known to significantly influence the overall erosion lifetime of plasma-facing materials ( $\mathrm{PFMs}$ ) as a result of the intense deposited energy during loss of plasma confinements [2]: (a) characteristics of incident plasma flow (i.e., particle type, kinetic energy, energy content, deposition time, and location) from the scrape-off-layer to the divertor plate; (b) characteristics of the vapor cloud that develops from the initial phase of energy deposition on target materials and its turbulent hydrodynamics; (c) generated-photon radiation and transport in the vapor cloud and to nearby regions; and (d) characteristics of plasma/solid/melt-layer interactions.

The comprehensive HEIGHTS computer simulation package was developed to evaluate in detail various effects of sudden high-energy deposition of different sources on target materials and to calculate erosion products and materials lifetime [3]. The package consists of several integrated models and codes that follow the beginning of the disrupting plasma from the scrape-off layer up to the transport of the eroded debris and splashed target materiais to nearby components. Factors that influence the lifetime of target materials and nearby components, such as loss of vapor-cloud confinement and vapor removal due to $M H D$ effects, damage to nearby surfaces due to intense vapor radiation, melt splashing, and brittle destruction of target materials, are also studied with the HEIGHTS package. The first part of the present work focuses mainly on modeling the erosion behavior of a metallic surface with a liquid layer subject to various internal and external forces during the energy-deposition phase, as well as the explosive erosion and characteristics of brittle-destruction erosion of carbon-based materials (CBMs). Lifetime predictions due to disruption erosion in a tokamak device are also presented.

Hydrogen isotope trapping and release by plasma facing and eroded/redeposited materials will influence fuel retention and recycling and plasma operation in future fusion reactors. Understanding particle and energy flow between PFMs and plasma is also necessary to optimize plasma performance, as well as to ensure safe and reliable reactor operation. Extensive studies of hydrogen diffusion and retention in fusion reactor materials have been carried out in recent years [4-6]. One important characteristic of the eroded materials is the porous nature of the redeposited layers. Erosion from plasmafacing and nearby components as a result of plasma instabilities is enough to form a redeposited layer on these components. Figure 2 schematically illustrates a typical layer 
structure of a porous material that may form as a result of dust deposition. The dust and debris of eroded materials may also be redeposited in different chemical forms (for example, $C T_{4}$ ). During reactor operation, therefore, new materials can be produced. The structure, chemical composition, transport, and retention properties of this material are quite different from the initial material. It is also very important to realize that the redeposited or codeposited layers can be porous and inhomogeneous, possibly resulting in more retained amount of tritium than in the initial PFM. Porosity is an important parameter that influences tritium buildup and release in candidate materials because of its nonlinear effect on the diffusion and retention of hydrogen isotopes. Diffusion in porous materials consists of three different and distinct diffusion processes: along grain boundaries, along microcrystallite boundaries, and diffusion in pure structure crystallites [7].
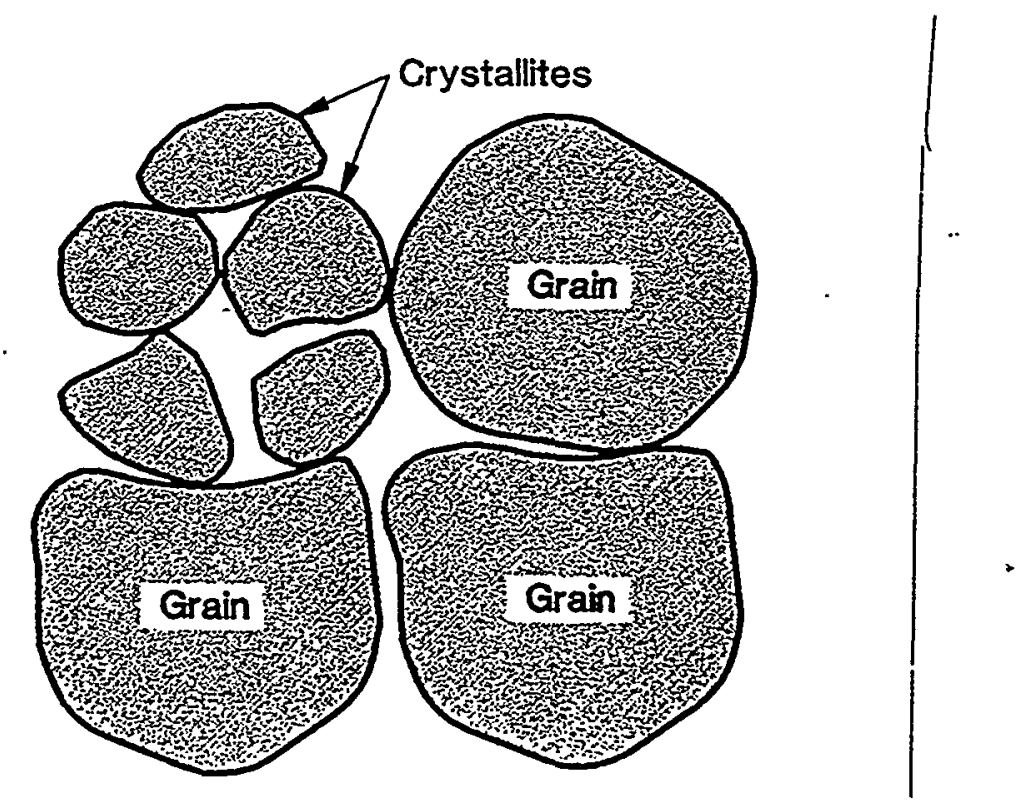

Fig. 2. Schematic view of porous structure of redeposited layers.

Comprehensive and reliable models of these phenomena do not fully exist and only few experimental data are available. Therefore, theoretical results of hydrogen isotopes retention and transport in PFM are not fully understood and do not always agree with available data. This discrepancy is especially due to the fact that diffusion in real 
candidate materials (graphites, $\mathrm{Be}, \mathrm{W}$ ) is much different (several orders of magnitude) than that of ideal homogeneous crystals, which is better described by theory. Existing models do not take into account, in a self-consistent manner, the strong inhomogeneous structure of real PFMs. The second part of the present work focuses on models of diffusion into nonhomogeneous porous materials implemented in TRAPS computer code. The effect of surface erosion during normal and abnormal operations on tritium diffusion and inventory is implemented in the TRICS code [7].

\section{Surface Erosion Mechanisms}

During the thermal-quench phase of a tokamak plasma disruption, part of the core plasma energy ( $>50 \%$ of total thermal energy) is delivered from the tokamak core to the SOL and then carried to the divertor plate by energetic plasma ion and electron fluxes. Therefore, the power load to the surface is very high, reaching hundreds of $\mathrm{GW} / \mathrm{m}^{2}$ and is capable of causing significant damage [1]. However, because of the developed vapor cloud of surface material in the early stages of a disruption above the divertor plate, the original surface is shielded from the incoming energy flux and the heat load onto the divertor plate surfaceis reduced significantly. Calculations with HEIGHTS predict that radiation power $\mathrm{W}_{\mathbf{S}}$ onto the divertor plate surface is $<10 \%$ of the original incident power because of the shielding effect [3].

Models for surface vaporization, material cracking and spallation, and liquid-metal ejection of melt layers have been developed for various erosion-causing mechanisms and implemented in the comprehensive HEIGHTS computer package [3]. Below are brief descriptions of some of these models and mechanisms used to study surface erosion and to predict component lifetimes.

\subsection{Erosion From Surface Vaporization}

Detailed physics of various interaction stages of plasma particles with target materials must be correctly modeled to evaluate the initial response of PFCs. Initially, the incident plasma particles of the disrupting plasma will deposit their energy at the surface of the target material. As a result, a vapor cloud of surface material quickly forms above the 
bombarded surface and in front of the incoming plasma particles. Depending on a range of parameters such as incident plasma power, magnetic field structure, geometrical considerations, vapor diffusion and motion, etc., the developed vapor cloud can significantly shield the original exposed areas from the incoming plasma particles and therefore further reduce surface damage.

To calculate the effectiveness of vapor-cloud shielding in protecting PFMs, detailed physics of plasma/vapor interactions have been modeled. The models include plasma particle slowdown and energy deposition in the expanding vapor, vapor heating, excitation, and ionization, and vapor-generated photon radiation. The detailed vapor motion above the exposed surface is calculated by solving the vapor MHD equations for conservation of mass, momentum, and energy under the influence of a strong magnetic field [8]. A significant part of the incident plasma kinetic energy is quickly transformed into vapor-generated photon radiation. Finally, multidimensional models for photon transport throughout the expanding vapor cloud have been developed to calculate the net heat flux that reaches the original disruption surface of plasma-facing components (PFCs), as well as the radiation heat load reaching various nearby components. It is the net heat flux reaching the surface that will further determine most of the response and the net erosion from surface vaporization, as well as from liquid splashing and brittle destruction of PFCs during these instabilities.

Figure 3 shows a typical time evolution of a tungsten-surface temperature, melt-layer thickness, and vaporization losses during a disruption for an incident plasma energy. of 10 $\mathrm{MJ} / \mathrm{m}^{2}$ deposited in a disruption time of $1 \mathrm{~ms}$, as predicted by HEIGHTS package [3]. An initial magnetic field strength of $5 \mathrm{~T}$ with an incident angle of $2^{\circ}$ is assumed in this analysis. The sharp initial rise in surface temperature results from the direct energy deposition of incident plasma particles at the material surface. The subsequent decrease in surface temperature was caused by the shielding effect of the eroded material accumulated above the target surface. The subsequent behavior of target is determined mainly by the energy flux from the emitted photon radiation in the vapor cloud, as discussed above, and by vapor-electron heat conduction. As more vapor accumulates above the surface, the vapor becomes more opaque to photon radiation and therefore less energy is transmitted to the target surface, in turn resulting in less splashing and vaporization. 


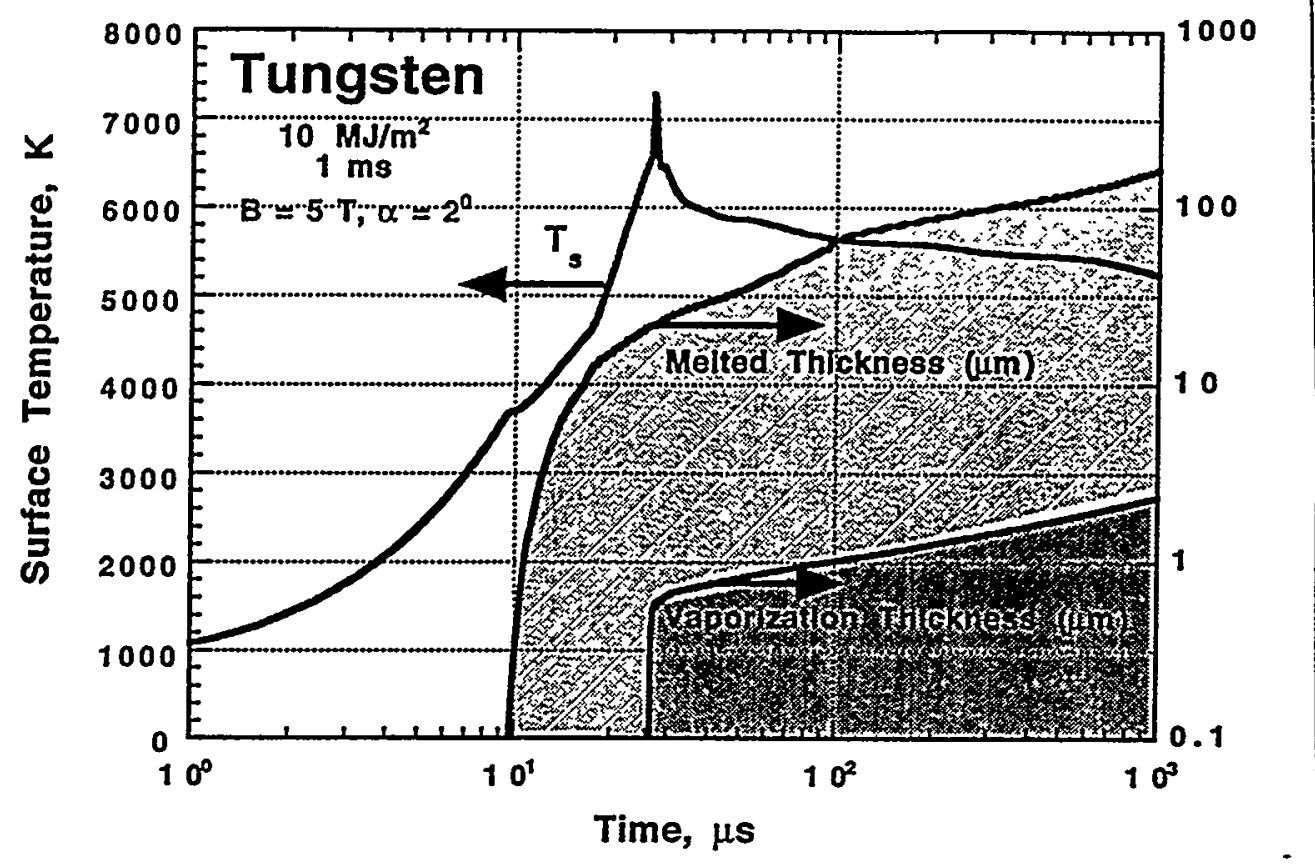

Fig. 3. Time evolution of tungsten temperature, melt layer, and vaporized thickness.

Calculation of photon radiation transport in this non-local thermodynamic equilibrium (non-LTE) vapor plasma is complex, tedious, and requires much computer time for reasonable accuracy. This is quite important because the results of this calculation will determine the net radiation power flux reaching the divertor surface and other nearby components. This net power flux will eventually determine PFC erosion lifetime. It follows from detailed calculations that the radiation power reaching the plate surface, $\mathrm{W}_{\mathrm{in}}$, is $\leq 10 \%$ of the incident total plasma power. There is a tendency for increasing radiation power at the plate surface with decreasing atomic number for lighter elements. Because the vapor cloud is more transparent in such cases, vapor shielding is less effective and therefore more radiation power reaches the target surface, causing more splashing [9]. The shape of the radiation power to the surface will also determine the spatial distribution of the damage profile [3]. 


\subsection{Splashing-Erosion of Melt Layers}

Radiation power reaching the target surface will result in both surface vaporization and ablation, i.e., mass loss in the form of macroscopic particles. Modeling predictions have shown that surface vaporization losses of metallic materials are small (only a few tens of micrometers deep) over a wide range of plasma conditions during shorter plasma instabilities. However, for liquid metals, surface ablation was predicted theoretically to be in the form of macroscopic metal droplets due to splashing of the molten layer [1]. Recent simulation experiments to predict erosion of candidate plasma-facing components during the thermal quench phase of a tokamak plașma disruption have also shown that erosion of metallic materials (such as $\mathrm{W}, \mathrm{Be}, \mathrm{Al}$, and $\mathrm{Cu}$ ) can be much higher than mass losses due only to surface vaporization. These mass losses depend strongly on experimental conditions such as level of incoming power, existence of a strong magnetic field, target inclination, etc. [3]. The mass losses are also found to be in the form of liquid-metal droplets with sizes ranging from $100 \AA$ to tens of micrometers leaving the target surface with velocities $V=10 \mathrm{~m} / \mathrm{s}$. Such ablation occurs as a result of splashing of the liquid layer due to several mechanisms [1]. Splashing erosion can occur due to boiling and explosion of gas bubbles in the liquid, absorption of plasma momentum, hydrodynamic instabilities developed in the liquid layer from various forces, runoff of melt layers over the structure, and mechanical vibration of the machine during the disruption. One main splashing mechanism results from the hydrodynamic instabilities developed in the liquid surface (such as Kelvin-Helmholtz and Rayleigh-Taylor instabilities). It was shown that Kelvin-Helmholtz instability can occur if the vapor plasma is not well-confined by the magnetic field and vapor flow occurs along the target surface. Another splashing mechanism is from volume bubble boiling, which usually occurs from overheating of the liquid metal above the vaporization temperature $T_{V}$, i.e., the temperature at which saturation pressure is equal to the outer pressure of the vapor plasma above the divertor plate surface. Therefore, the erosion energy is roughly equal to the sum of the thermal energy (required to heat the liquid above a certain temperature, i.e., melting temperature for hydrodynamic instabilities and vaporization temperature for bubble boiling), melting energy (i.e., heat of fusion), and kinetic energy of the droplets. 
The kinetic energy of the splashed droplets is determined from the surface tension of the liquid metal.

To correctly predict melt-layer erosion, a four-moving-boundaries problem is solved in the HEIGHTS package [8]. The front of the vapor cloud, generated from the initial plasma power deposition, is one moving boundary determined by solving vapor hydrodynamic equations. The second moving boundary due to surface vaporization of the target is calculated from target thermodynamics. Immediately following the surface vaporization front is a third moving boundary due to the melt-splashing front. Finally, the fourth moving boundary is at the liquid/solid interface and further determines the new thickness of the melt layer. These moving boundaries are interdependent, and a selfconsistent solution must link them dynamically and simultaneously. It is the third moving boundary (the liquid splashing front), however, that determines the extent of metallic PFC erosion and lifetime due to plasma instabilities. The SPLASH code (part of the HEIGHTS package) calculates mass losses by using a splashing-wave concept as a result of each erosion-causing mechanism [3]. Thus, total erosion can be calculated from the sum of all possible erosion mechanisms.

\subsection{Macroscopic Erosion of Carbon-Based-Materials}

Nonmelting materials such as graphite and CBMs have also shown large erosion losses significantly exceeding that from surface vaporization. Models were developed to evaluate erosion behavior and lifetime of CBMs of plasma-facing and nearby components due to brittle destruction during plasma instabilities [3]. The macroscopic erosion of CBMs depends on three main parameters: net power flux to the surface, exposure time, and threshold energy required for brittle destruction. The required energy for brittle destruction is critical in determining the net erosion rate of CBMs and is estimated to be $\approx 10 \mathrm{~kJ} / \mathrm{g}$, or $\approx 20 \mathrm{~kJ} / \mathrm{cm}^{3}$. Therefore, for a net power flux to the material surface during the disruption of $\approx 300 \mathrm{~kW} / \mathrm{cm}^{2}$, the deposited energy for a time of $1 \mathrm{~ms}$ is $\approx 0.3 \mathrm{~kJ} / \mathrm{cm}^{2}$, resulting in net erosion of $\approx 150 \mu \mathrm{m}$ per disruption. This is much higher than that predicted from pure surface vaporization of $\approx 10 \mu \mathrm{m}$ per disruption for CBMs [3]. A sacrificial coating/tile thickness $\approx 1 \mathrm{~cm}$ thick would last fewer than 70 disruptions. Again, this is far fewer than 
the current expectation of several hundred disruptions during the reactor lifetime. Longer disruption times can also significantly reduce disruption lifetime. Therefore, more relevant experimental data and additional detailed modeling are needed to evaluate the erosion of CBMs, which strongly depends on the type of carbon material.

\section{Droplets and Macroscopic Shielding Concept}

Complete and accurate calculation of mass losses during plasma instabilities requires a full MHD description of the vapor media near the target surface; the media consist of a mixture of vapor and droplets moving away from surface. Photon radiation power from the upper regions of the vapor cloud will then be absorbed by both the target surface and the droplet cloud, resulting in the surface vaporization of both target and droplet surfaces. Therefore, in such a mixture of erosion products, further screening of the original target surface occurs because of the splashed droplets or macroscopic CBM debris. This has the effect of reducing photon radiation power to the target surface. Such screening can be called "droplet shielding" in an analogy to the vapor shielding effect [9]. Features of this droplet shielding and its influence on total mass loss are calculated for the cases of volume bubble boiling with homogeneous velocities of droplets in momentum space, and in the case of Rayleigh-Taylor instability with droplets that move normal to the surface preferentially [9].

To summarize the simulation results, due to overheating of the divertor plate surface, macroscopic particles and droplets are ejected/splashed upstream and away from the surface. These particles then absorb some part of the incoming vapor radiation. The net fraction of radiation power reaching the divertor plate surface is determined only by the ratio of vaporization to splashing energies. The distance at which macroscopic particles are completely vaporized is calculated to be about 100 times the initial radius of droplets. Because the initial droplet radius is small $(\leqslant 10 \mu \mathrm{m})$, the droplets completely vanish at distance $\mathrm{L} \leq 1 \mathrm{~cm}$. Therefore, the mixture of vapor and macroscopic particles exists only very near the divertor plate surface. Because the vaporization energy is much higher than the energy required for splashing/destruction, this means that most radiation power from the upper vapor cloud is expended in vaporization. Therefore, despite the large initial

splashing erosion, total erosion of the divertor plate is defined only by vaporization 
losses, including both divertor plate vaporization and macroscopic-particle vaporization. Again, this is true only if both the vapor cloud and the splashed droplets are well-confined in front of the incoming disrupting plasma.

\section{Tritium Transport Mechanisms in the 2-D TRÁPS Model}

Two- and three-dimensional effects of a PFM porous-structure and the redeposited dust and debris of these materials on tritium diffusion and inventory were previousely discussed in more detail [7]. An important feature of this model is enhanced in the 2-D TRAPS code, i.e., modeling of diffusion in grain-structured materials. It is known that tokamak candidate materials ( $\mathrm{C}, \mathrm{Be}, \mathrm{W}$ ) will consist of grains with size, $L_{g}$, in the order of a few micrometers and are separated from each other by an intergrain substrate with width, $d_{g}$, where $d_{g} \approx 0.1 L_{g}$. The grains in turn consist of crystallites with size $L_{c}$ on the order of $50-100 \AA$, that are also separated from each other by intercrystallite substrates with width $d_{c} \approx 0.1 L_{c}$, as schematically illustrated in Fig. 4 [9].

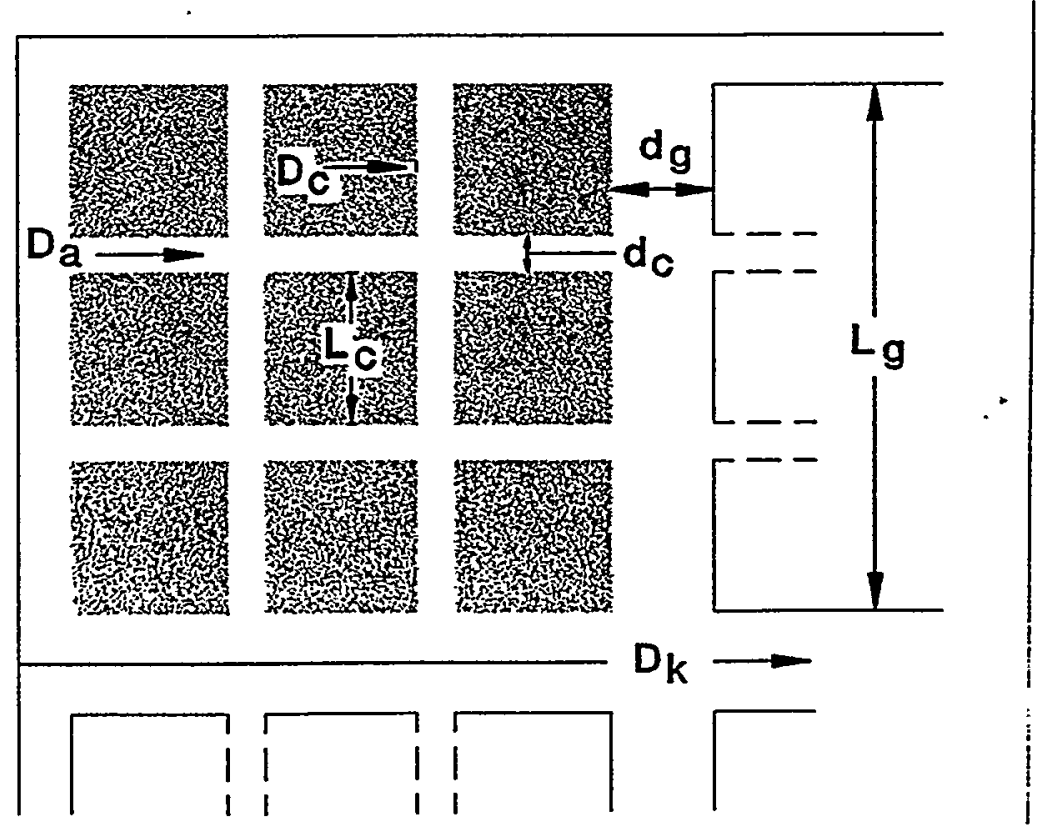

Fig. 4. Numerical simulation model of a porous structure used in TRAPS. 
The diffusion coefficients of both the intergrains substrate, $D_{k}$, and intercrystallite substrate, $D_{a}$, are much greater than the diffusion coefficient, $D_{c}$, of the crystallites, which is similar to that of an ideal crystal. This means that implanted plasma particles $(T, D)$ will diffuse mainly along these substrates. After saturation of the substrates, the implanted gas diffuses into the crystallites. Because of the small size, $L_{c}$, of crystallites, the diffusion time into the crystallites, $\tau_{c}$, becomes comparable to or smaller than the diffusion time along the substrates:

$$
\tau_{c}=\frac{\mathrm{L}_{c}^{2}}{\mathrm{D}_{\mathrm{c}}} \approx \frac{\mathrm{L}_{\mathrm{g}}^{2}}{\mathrm{D}_{\mathrm{k} / \mathrm{a}}}, L_{c} \ll L_{g}, D_{k} \approx D_{a}
$$

To describe diffusion in such a medium, direct numerical modeling requires much computer time. Therefore, we use a set of equations that regards this inhomogeneous discrete system of grains and crystallites as a continuous medium but nevertheless takes into account the inherent discrete structure, i.e., existence of grains, crystallites, and intergrain/intercrystallite substrates [7].

The full set of equations describing such a system is very complicated; therefore, for illustration of this work, only a simplified set of equations is given, ignoring the diffusion in the vertical intercrytallite channels (along $\mathrm{Y}$ axis) and in the horizontal intergrain channels (along $\mathrm{X}$ axis). In this model, crystallites are also regarded as spheres with an effective radius close to that of $L_{c}$. These set of equations are given by [7]:

$$
\begin{aligned}
& \frac{\partial n_{k}}{\partial t}=D_{k}^{*} \frac{\partial^{2} n_{k}}{\partial y^{2}}-S_{k a}+S_{a k}-S_{k}^{+}+S_{k c}^{-} \\
& \frac{\partial n_{a}}{\partial t}=D_{a}^{*} \frac{\partial^{2} n_{k}}{\partial x^{2}}-S_{a c}+S_{c a}-S_{a t}^{+}+S_{a s}^{-} \\
& \frac{\partial n_{c}}{\partial t}=D_{c}^{*} \frac{1}{r^{2}} \frac{\partial}{\partial r} r^{2} \frac{\partial n_{c}}{\partial r}+S_{a c}-S_{c t}^{+}+S_{c t}^{-},
\end{aligned}
$$


where subscripts $k, a, c$ are those relating to intergrains substrate, intercrystallites substrate, and the crystallites, respectively, and $D_{\alpha},(\alpha=k, a, c)$ is the corresponding diffusion coefficient defined from $l_{\alpha}$ and $\tau_{\alpha}$, i.e., the corresponding path length and collision time of medium $\alpha$ respectively, and $n_{\alpha s}$ is maximum dissolved density. The linear number of crystallites in one grain is given as $N_{c}=L_{g} / L_{c}$. Fluxes in, $\mathrm{S}_{\alpha x}^{+}$, and out, $S_{\alpha x}^{-},(x=k, a, c)$ of intergrains, intercrystallites, crystallites, and traps can be given by certain distribution functions $[7,10]$. Results of such modeling indicated that three distinctive regimes exist with three effective diffusion coefficients $\left(D_{1}, D_{2}, D_{3}\right)$ corresponding to diffusion along grain boundaries, diffusion along crystallite boundaries, and into crystallites, taking into account the 2-D structure of the channels. The analysis indicates strong dependence on the porous structure parameters and dimensions [7].

To model tritium behavior in the original PFM as a result of erosion, the selfconsistent computer model TRAPS is used [7]. The code can include up to four different tritium trapping-sites with different spatial and trapping energy distribution in the coating as well as in the substrate materials. Inhomogeneous trap distribution is necessary to account for traps created by the implanted flux, which is near the surface region, and those traps created by neutron irradiation distributed throughout the coating and the substrate structure.

Surface erosion in the TRICS code is implemented in two ways [7]: continuous erosion rate as a result of normal operation, and pulsed erosion rate due to abnormal events. Continuous erosion rate is due mainly to physical and chemical sputtering of the surface coating material. As a result of the continuous erosion, the surface location is a moving coordinate with time. The surface temperature will continue to decrease because the thickness of the material is decreasing and the incident heat flux is presumed to be time-independent. Figure 5 illustrates these processes [7]. At each time-step, the coating thickness is decreased by a certain amount, depending on the erosion rate. The decrease in the surface temperature as a result will have two main effects, i.e., reduction in recombination flux at the surface, and a resulting increase in permeation flux at the coolant side. The reduction in the coating thickness will also result in a reduction in tritium inventory and in the diffusing flux under typical tokamak conditions [7]. 


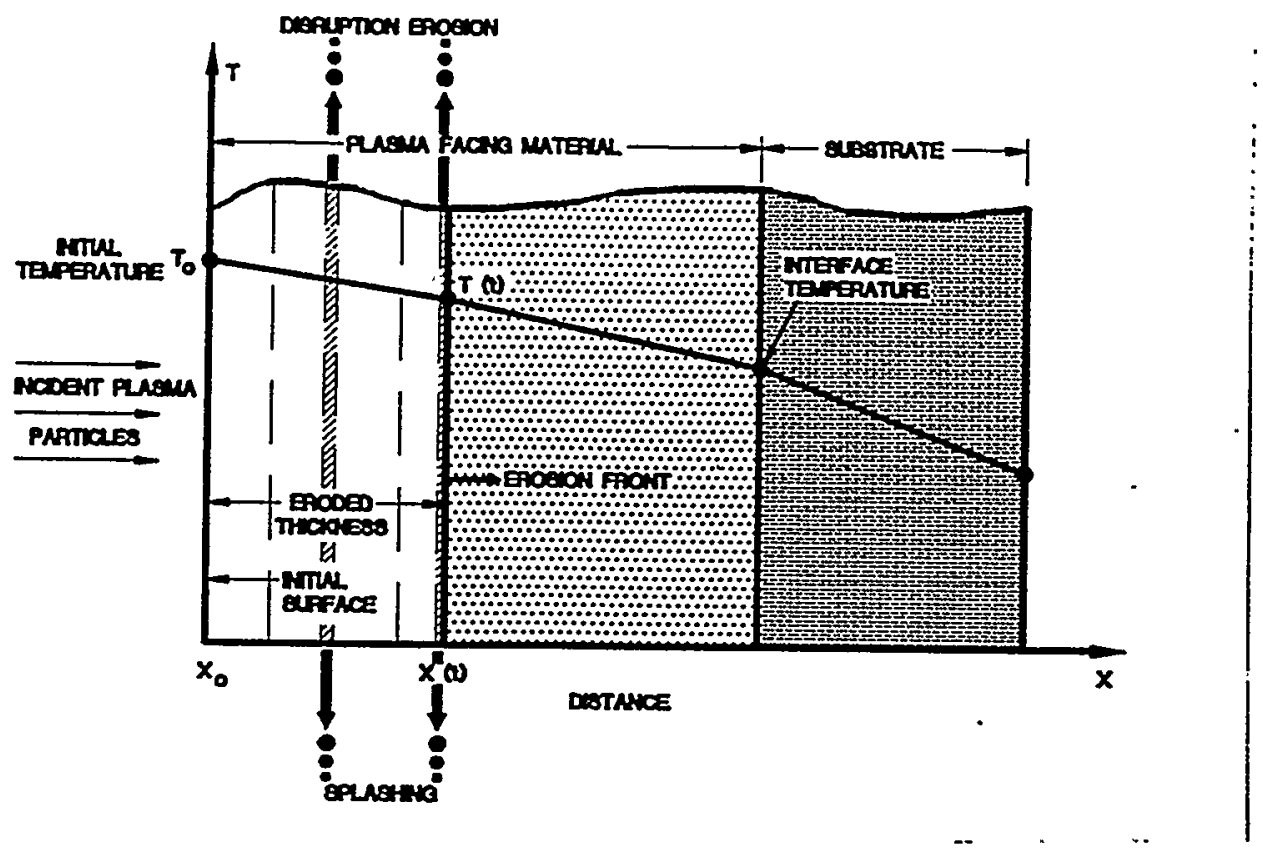

Fig. 5. Mlustration of erosion processes and moving surface coordinates.

In addition, the TRICS code can account for the effect of sudden erosion due to abnormal events such as plasma disruptions and vertical displacement events. In such cases a surface layer of a certain thickness is removed with all its tritium inventory and trap concentrations. The tritium contained in the eroded materials will decrease as time passes because more tritium is diffusing to the coolant side as a result of reducing the total coating thickness. The code can also model tritium behavior in eroded or splashed material, as a result of plasma instabilities, and its redeposition on colder nearby surfaces. Future work will focus on complete and more accurate estimates of tritium diffusion and inventory for conditions resembling that of the tokamak Demo design.

\section{Conclusions}

The effect of porosity of redeposited dust and debris materials on tritium diffusion and inventory in such materials is quite important. The 2- and 3-dimensional effects of the 
porous structure of the redeposited dust and debris of PFMs (carbon, beryllium...) as well as that from plasma-spraying techniques or from original as-fabricated materials must be taken into account in the numerical models describing gas diffusion and gas release in porous two-level structural materials. Specifically designed laboratory experiments in which the dust and debris of candidate PFMs are produced and studied are required to correctly predict tritium behavior in these materials. Preliminary models of diffusion in porous materials, including diffusion along grain boundaries, intercrystallite substrates, and into crystallites, were developed. TRAPS calculations show that tritium filling of a porous material having a certain solubility and number of traps has the form of "fillingwave" that takes on a one-dimensional nature at times exceeding these of characteristic times of diffusion into separate grains and crystallites. TRAPS also takes into account other important processes such as recombination rates of atoms on material and pore surfaces and the existence of open and closed pores. Future work will evaluate and assess the total tritium inventory in porous materials as a function of reactor operating conditions. The TRICS code was developed to study the effect of surface erosion on tritium behavior in the original plasma-facing materials. Erosion generally will increase tritium concentrations in the coolant and in the eroded and splashed debris due to permeation and trapping.

\section{Acknowledgment}

Work is supported by the U.S. Department of Energy, Office of Fusion Energy Science, under Contract 20-31-109-Eng-38.

\section{References}

1. Hassanein, A. et al. (1998) Fusion Eng. Des. 39 \& 40, 201.

2. Hassanein, A. and Konkashbacv, I. (1996) J. Nucl. Mater., 233-237, 713.

3. Hassanein, A. and Konkashbaev, I. (1999) J. Nucl. Mater. 273, 326.

4. Andrew, P.L. and Pick. M.A. (1994) J. Nucl. Mater. 212-215, 111.

5. Federici, G., et al. (1995) Fusion Eng. and Design 28, 136.

6. Baskes, M.I. (1980) J. Nucl. Mater. $85 \& 86,318$.

7. Hassanein, A. et al. (1998) J. Nucl. Mater. 258-263, 295.

8. Hassanein, A. (1996) Fusion Technol. $30,713$.

9. Hassanein, A. et al. (2000) to be published in Fusion Eng. \& Design.

10. Konkashbaev, I. et al. (1997) Fusion Technology, C. Varandas and F. Serra, eds. 1803. 